\title{
Application of a parallel genetic algorithm to the global optimization of medium-sized Au-Pd sub-nanometre clusters ${ }^{\star, \star \star}$
}

\author{
Heider A. Hussein ${ }^{1,2}$, Ilker Demiroglu ${ }^{1}$, and Roy L. Johnston ${ }^{1, a}$ \\ ${ }^{1}$ School of Chemistry, University of Birmingham, Birmingham B15 2TT, UK \\ ${ }^{2}$ Department of Chemistry, College of Science, University of Kufa, Najaf, Iraq
}

Received 31 May 2017 / Received in final form 30 June 2017

Published online 12 February 2018

(C) The Author(s) 2018. This article is published with open access at Springerlink.com

\begin{abstract}
To contribute to the discussion of the high activity and reactivity of $\mathrm{Au}-\mathrm{Pd}$ system, we have adopted the BPGA-DFT approach to study the structural and energetic properties of medium-sized Au$\mathrm{Pd}$ sub-nanometre clusters with 11-18 atoms. We have examined the structural behaviour and stability as a function of cluster size and composition. The study suggests $2 \mathrm{D}-3 \mathrm{D}$ crossover points for pure Au clusters at 14 and 16 atoms, whereas pure Pd clusters are all found to be 3D. For Au-Pd nanoalloys, the role of cluster size and the influence of doping were found to be extensive and non-monotonic in altering cluster structures. Various stability criteria (e.g. binding energies, second differences in energy, and mixing energies) are used to evaluate the energetics, structures, and tendency of segregation in sub-nanometre $\mathrm{Au}-\mathrm{Pd}$ clusters. HOMO-LUMO gaps were calculated to give additional information on cluster stability and a systematic homotop search was used to evaluate the energies of the generated global minima of monosubstituted clusters and the preferred doping sites, as well as confirming the validity of the BPGA-DFT approach.
\end{abstract}

\section{Introduction}

Due to the environmental and energy challenges facing the world, research in catalysis is particularly important. Nanometallic catalysts, in particular, often show superior performance compared to their bulk counterparts $[1,2]$. These catalysts, which are widely used in the chemical industry, have motivated experiments on new materials on the nanoscale with high catalytic activity and/or selectivity [3-5] and have inspired the development of computational methods for predicting new catalyst candidates and optimizing their efficiency $[6,7]$.

Although extensive research indicates that platinum and platinum-based nanostructures exhibit exceptional electrocatalytic activity, for example in direct alcohol fuel cells $[8,9]$ their applications are somewhat limited due to the rarity of $\mathrm{Pt}$ in the earth's crust and its consequent high cost. This has motivated researchers to look for alternative metals (or alloy systems) to replace Pt, ideally keeping the high performance but at lower cost [10-12]. The much higher natural abundance of $\mathrm{Pd}$ relative to $\mathrm{Pt}$

\footnotetext{
* Contribution to the Topical Issue "Shaping Nanocatalysts", edited by Francesca Baletto, Roy L. Johnston, Jochen Blumberger and Alex Shluger.

${ }^{\star}$ Supplementary material in the form of one PDF file available from the Journal web page at https://doi.org/10.1140/ epjb/e2017-80314-2.

a e-mail: r.1.johnston@bham.ac.uk
}

has led to researchers fabricating nanostructures based on $\mathrm{Pd}[7,11]$. Pd-based bimetallic nanostructures have also been used extensively, not only in the application to the direct alcohol fuel cells (as the cathodic catalyst) [11] but also in many high-tech fields, due to their interesting magnetic and electronic properties, as well as their catalytic properties $[2,8,13,14]$.

Changing the chemical order and composition of the bimetallic nanostructures can enhance and enable tuning of their catalytic properties [15,16]. The chemical order effect can lead to control of the catalytic properties (e.g. modifying relevant activation energy barriers) by tuning the energy and spatial distribution of electrons at the surface of the clusters [2]. There are also economic reasons for mixing two metals, such as adding low cost metals (e.g. $\mathrm{Co}, \mathrm{Cu}$, and $\mathrm{Ni}$ ) to balance the high cost of noble metals. The enhancement of catalytic performance and possible discovery of unique properties is still the main force driving the designing of novel nano-catalysts and sub-nanometre cluster catalysts. The $\mathrm{Pd}-\mathrm{Au}$ bimetallic system, for example, exhibits high durability and catalytic activity for many interesting chemical reactions, such as the electro-oxidation of ethanol $[17,18]$, the Suzuki coupling reaction [19], and the oxygen reduction reaction [20].

The catalytic activity and selectivity of sub-nanometreand nano-clusters are also strongly affected by the size of the cluster and its electronic distribution. The origin of size effects are still ambiguous [21], however, they are 
usually attributed to the changing surface-area-to-volume ratio and the number and nature of exposed facets/surface sites [22-24].

Gas-phase sub-nanometre clusters are simplified models that can test a system's suitability for specific applications, at a reasonably high level of theory, before dealing with more complex systems. However, even the optimisation of gas-phase structures is not a trivial task. There is usually little energy separation between many competitive isomers of metallic clusters [25], which may explain the challenges that face experimentalists in determining the preferred isomer [26,27]. In addition, charge-neutral clusters are more difficult to investigate experimentally compared with anions and cations, as most characterizations rely on mass spectrometry measurements, which creates a clear difficulty in separating and probing different size of neutral clusters $[28,29]$.

In spite of the early investigations of neutral $\mathrm{Au}_{2}$ [30] and $\mathrm{Au}_{3}$ [31]. It is well known that experimental investigations of medium sized neutral Au clusters are limited for the reasons given above. Hence, a combination of experimental techniques with theoretical calculations has been used effectively: for example, Fielicke and co-workers [28] reported the gas-phase structures of neutral $\mathrm{Au}_{7}, \mathrm{Au}_{19}$, and $\mathrm{Au}_{20}$ clusters tagged with $\mathrm{Kr}$ atoms. The case of $\mathrm{Pd}$ is similar as, unlike the smaller Pd clusters, studies of medium sized clusters are relatively few and are the object of some controversy in terms of identifying the structural characteristics [32]. Turning our attention to medium-sized $\mathrm{Au}-\mathrm{Pd}$ nanoalloys, a comprehensive study of structural motifs for all compositions have, to our knowledge, not been investigated before. However, Au-doped Pd clusters [33], (1:1) compositions [34], and nuclearities lower than 14 atoms [35] have previously been studied theoretically.

In the $\mathrm{Au}-\mathrm{Pd}$ system, doping an atom of one metal into a pure cluster of the other metal often yields clusters with non-identical structures and properties [35-37]. This can increase the difficulty in finding global minima, in addition to the permutational isomers (homotops) effect [38]. There is additional complexity introduced by multi-directional bonding, unrestrained bond orders, and fluxional behaviour as a result of electron delocalization. In addition, alloying $\mathrm{Pd}$ with $\mathrm{Au}$ modifies the lattice distance between host atoms and the low dimensionality of $\mathrm{Au}$ could influence the spatial arrangement of $\mathrm{Pd}$ atoms $[39,40]$.

The DFT-based Birmingham Parallel Genetic Algorithm (BPGA-DFT) computational approach has been successfully applied to search for low-lying isomers for various sub-nanometre cluster systems. Applications include: pure clusters $\mathrm{Ir}_{10}-\mathrm{Ir}_{20}$ [41], $\mathrm{Rh}_{4}-\mathrm{Rh}_{6}$ [42], $\mathrm{Au}_{4}-\mathrm{Au}_{10}$, $\mathrm{Pd}_{4}-\mathrm{Pd}_{10}$ [37], $\mathrm{Ru}_{3}-\mathrm{Ru}_{12}$ and $\mathrm{Pt}_{3}-\mathrm{Pt}_{10}$ [43]; and bimetallic clusters $(\mathrm{AuRh})_{4-6}$ [42]. (AuPd $)_{4-10}$ [37], (AuIr $)_{4-6}$ [44], and $(\mathrm{RuPt})_{3-8}$ [43] as well as surface-supported $[37,44]$ clusters.

In this context, we present here a computational study of the structural properties of binary sub-nanometre $\mathrm{Au}^{-}$ Pd clusters, including a comparison to the pure clusters. Using the BPGA-DFT approach, the lowest energy structures in the size range 11-18 atoms were calculated for all compositions. This work also sheds some light on the energetics of these clusters and the underlying mechanisms of mixing in binary metallic systems on the sub-nanometre and nanoscale. Our findings for the $\mathrm{Au}-\mathrm{Pd}$ system should provide valuable information for $\mathrm{Au}-\mathrm{Pd}$ catalysts and for further theoretical and experimental investigations.

\section{Methodology}

The Birmingham Parallel Genetic Algorithm (BPGADFT) approach [41,45] was applied to investigate (at the DFT level) the lowest energy structures of $\mathrm{Au}-\mathrm{Pd}$ sub-nanometre clusters with total number of atoms $N=$ 11-18, as well as pure $\mathrm{Au}_{N}$ and $\mathrm{Pd}_{N}$ clusters. BPGADFT is an open-source genetic algorithm [45], which is a parallel extension of the Birmingham Cluster Genetic Algorithm (BCGA), a genetic algorithm for locating the global minima of small metal clusters directly at the DFT level [46].

Instead of generations, BPGA-DFT employs a pool methodology to evaluate structures in parallel. In each run, multiple BPGA instances are implemented, and in each instance, a set of processes are run in parallel and independently $[47,48]$. Initially, the pool population is formed by generating a number of random isomers [41]. The ten generated structures forming the initial pool are geometrically relaxed by local DFT energy minimization [47]. Once minimized structures are generated, the genetic algorithm crossover and mutation operations are applied to members of the population.

The clusters are selected for either crossover or mutation. The crossover operation involves selecting a pair of clusters from the pool, using the tournament selection method, based on a fitness criterion, where the fittest isomers (those with the lowest DFT energies) are more likely to be selected for crossover. Offspring clusters are then generated using the cut-and-splice method introduced by Deaven and Ho [49]. There are two mutation operations, in which a single cluster is randomly selected and either randomly chosen atoms are displaced or (for bimetallic clusters) the positions of a randomly chosen pair of non-identical atoms are swapped. After crossover and mutation, the structures are locally energy-minimized at the DFT level. The newly generated structures are then compared energetically with existing structures in the pool and the highest energy isomers are replaced by any lower energy isomers among the set of offspring and mutants.

All the local energy minimizations mentioned above were conducted with gamma-point DFT calculations employing the Vienna ab initio Simulation Package (VASP) code [50]. Projected-augmented wave (PAW) pseudopotentials were used, with the (GGA) PerdewBurke-Ernzerhof (PBE) exchange correlation functional [51,52]. A plane-wave basis set was implemented including spin polarization. The plane wave cut-off energy was truncated at $400 \mathrm{eV}$. Methfessel-Paxton smearing, with a sigma value of $0.01 \mathrm{eV}$, was implemented to improve convergence [53]. 
For pure $\mathrm{Au}$ and $\mathrm{Pd}$ and mixed $\mathrm{Au}-\mathrm{Pd}$ clusters, the stability of each cluster, relative to neighbouring sizes, is indicated by the second difference in energy $\left(\Delta_{2} E\right)$ which is given by:

$$
\Delta_{2} E=E\left(A_{N+1}\right)+E\left(A_{N-1}\right)-2 E\left(A_{N}\right)
$$

where $E\left(A_{N}\right)$ corresponds to the energy of the $N$-atom cluster and $E\left(A_{N+1}\right)$ and $E\left(A_{N-1}\right)$ are the neighbouring clusters, with one atom more and one atom less, respectively.

The effect of mixing Au with $\mathrm{Pd}$ atoms in nanoalloys can be evaluated by calculating the mixing or excess energy $(\Delta)$ which is given by:

$$
\Delta=E_{\left(\mathrm{Au}_{m} \mathrm{Pd}_{n}\right)}-m \frac{E_{\left(\mathrm{Au}_{N}\right)}}{N}-n \frac{E_{\left(\mathrm{Pd}_{N}\right)}}{N}
$$

where $m$ and $n$ are the numbers of $\mathrm{Au}$ and $\mathrm{Pd}$ atoms, respectively, $E_{\left(\mathrm{Au}_{m} \mathrm{Pd}_{n}\right)}$ is the total energy of the nanoalloy $\mathrm{Au}_{m} \mathrm{Pd}_{n}$ whereas $E_{\left(\mathrm{Au}_{N}\right)}$ and $E_{\left(\mathrm{Pd}_{N}\right)}$ are the energy of pure metal clusters of $\mathrm{Au}$ and $\mathrm{Pd}$, respectively, of the same size $(N=m+n)$.

The average binding energy per atom $\left(E_{b}\right)$ is given by:

$$
E_{b}=-\frac{1}{N}\left[E_{\left(A u_{m} P d_{n}\right)}-m E_{(A u)}-n E_{(P d)}\right]
$$

where $E_{(A u)}$ and $E_{(P d)}$ are the electronic energies of single $\mathrm{Au}$ and $\mathrm{Pd}$ atoms, respectively.

The homotops (inequivalent permutational isomers) [38] are evaluated using:

$$
\Delta E=E_{h o m}-E_{G M}
$$

where $\Delta E$ is the relative energy of the proposed homotop and $E_{h o m}$ and $E_{G M}$ are the electronic energies of a particular homotop and the lowest energy isomer (global minimum) of the cluster, respectively.

Ignoring the symmetry, the number of homotops is given by:

$$
{ }^{N} P_{A u, P d}=\frac{N !}{m ! n !}=\frac{N !}{m !(N-m) !}
$$

where $N$ is the total number of atoms, $m$ is the number of $\mathrm{Au}$ atoms, and $n$ is the number of $\mathrm{Pd}$ atoms.

\section{Results and discussions}

\subsection{Structures}

\subsubsection{Au clusters}

The putative global minima for pure Au clusters $11 \leq$ $N \leq 18$, are shown in Figure 1 and their energies, coordinates, and point groups are listed in Table S1 (see the Supporting Information).

The lowest energy structures obtained for $N=11-$ 13 and $15 \mathrm{Au}$ clusters have planar (2D) configurations. The clusters deviate from planar to 3D structures at
$N=14$ and for $16-18$ atoms. The exact 2D-3D transition point for neutral Au clusters is disputed theoretically and experimental evidence is scarce. Theoretical predictions of the $2 \mathrm{D}-3 \mathrm{D}$ crossover point have previously ranged from $N=7-14$ atoms [54-57]. This range is consistence with the evolution of structure-symmetry for Au clusters reported here. The smallest 3D ground-state structure was predicted previously to be $\mathrm{Au}_{10}$ by David and co-workers [58], using the second-order Møller-Plesset perturbation theory (MP2) method. Employing hybrid DFT, Zanti and Peeters [35] arrived at the same conclusion showing a 3D structure for $N=10$. This, however, disagreed with the $2 \mathrm{D} \mathrm{Au}_{10}$ structure recently obtained by us [37] and previously by different research groups using semi-local density functional theory (DFT) [59] and coupled cluster singles doubles and perturbative triples $[\operatorname{CCSD}(\mathrm{T})]$ calculations $[60,61]$. Recently, Johansson et al., using a genetic algorithm and meta-GGA DFT, assigned a 3D structure as the GM structure at $N=12$. However, they also suggested two isoenergetic structures (2D and $3 \mathrm{D}$ ) for $N=11$ atoms [62].

Three generic structure types can be identified for $\mathrm{Au}$ clusters (see Fig. 2): (i) a 2D close packed planar layer (analogous to the (111) face of fcc bulk gold) for $N=11$ 13 and 15; (ii) a condensed flattened cage structure for $N=14$ and 16 clusters; (iii) a pseudo-spherical hollow cage structure for $N=17-18$. To explain the difference between the flattened and hollow cage structures, we can compare the predicted structures of $\mathrm{Au}_{16}$ and $\mathrm{Au}_{17}$, which are shown in Figure 3. For $\mathrm{Au}_{16}$ (flattened cage), the dimensions of the shortest two internal axes are $0.3 \mathrm{~nm}$ and $0.7 \mathrm{~nm}$. This cage could accommodate a small atom (e.g. $\mathrm{H}, \mathrm{He}, \mathrm{Ne}, \mathrm{O}$ or $\mathrm{F}$ ). However, for $\mathrm{Au}_{17}$ (hollow cage), the dimensions are both $0.6 \mathrm{~nm}$ and the cage could accommodate larger atoms, even an extra $\mathrm{Au}$ atom.

The planar structures obtained for the global minima of $\mathrm{Au}_{N}(N=11-13$ and 15$)$ clusters agree with the findings of $\mathrm{Fa}$ et al. [63] concerning $\mathrm{Au}_{11}$ and $\mathrm{Au}_{12}$. However, a difference is observed for $\mathrm{Au}_{13}$ and $\mathrm{Au}_{15}$, which are predicted to be 3D by Fa. The nearest low-lying isomer to our $2 \mathrm{D} \mathrm{Au}_{13}$ global minimum is predicted to be $3 \mathrm{D}$, with an energy $0.5 \mathrm{eV}$ higher than the GM. The lowest-energy structures of $\mathrm{Au}_{14}$ shows flattened cage structures, as previously reported $[63,64]$. The competitive isomer for the $3 \mathrm{D}$ GM of $\mathrm{Au}_{14}\left(\mathrm{C}_{2 \mathrm{~V}}\right)$ is also $3 \mathrm{D}\left(\mathrm{C}_{2 \mathrm{~V}}\right)$, with a relative energy of only $9 \mathrm{meV}$. The lowest-energy structure of $\mathrm{Au}_{15}$ is a $2 \mathrm{D}$ close packed layer, with $\mathrm{C}_{2 \mathrm{~V}}$ symmetry. The second most stable isomer is $3 \mathrm{D}\left(\mathrm{C}_{2 \mathrm{~V}}\right)$, with relative energy $0.19 \mathrm{eV}$. Having a flattened cage structure and $\mathrm{C}_{2 \mathrm{v}}$ symmetry, $\mathrm{Au}_{16}$ is similar to the case of $\mathrm{Au}_{14}$, as previously reported [64]. In contrast, anionic $\mathrm{Au}_{16}$ has been reported to adopt a hollow cage structure [65].

The structural transition from flattened cage to hollow cage configurations occurs at $N=17$. The lowest energy structure we have obtained for $\mathrm{Au}_{17}$ is similar to what has been reported for neutral [64] and anionic [65] $\mathrm{Au}_{17}$, showing a pseudo-spherical hollow cage structure with $\mathrm{C}_{2 \mathrm{~V}}$ symmetry. The nearest competitive isomer to the GM is also another hollow-cage structure, with a relative energy of $0.08 \mathrm{eV}$ and $\mathrm{C}_{1}$ symmetry. The hollow cage structure $\left(\mathrm{D}_{4 \mathrm{~d}}\right)$ observed for $\mathrm{Au}_{18}$ is different from the 


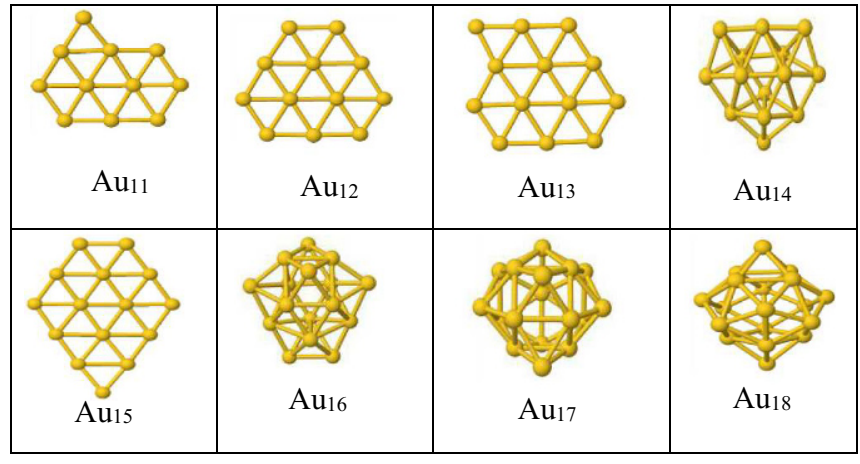

Fig. 1. Putative global minimum structures for $\mathrm{Au}_{N}$ clusters, $N=11-18$.

hollow cage structure reported by Bulusu and Zeng [64], though the latter agrees with the energetically competitive isomer that we have found, which is only $0.08 \mathrm{eV}$ higher in energy than the GM with $\mathrm{C}_{\mathrm{S}}$ symmetry.

\subsubsection{Pd clusters}

Figure 4 shows the putative global minima for Pd clusters: their energies, coordinates and point groups are listed in Table S1 (see the Supporting Information).

Similar to small Pd clusters [37], the lowest energy structures obtained here for medium-sized Pd clusters are all 3D. As for pure Au clusters, the structural motifs adopted by $\mathrm{Pd}$ clusters are size-dependent; for mediumsized Pd clusters, a structural transition occurs at $N=15$ atoms from bilayer structures to filled cage structures.

Global minima for the gas phase $\mathrm{Pd}_{11}, \mathrm{Pd}_{13}$ and $\mathrm{Pd}_{14}$ clusters are found to be distorted hexagonal bilayer (HBL) structures with $\mathrm{C}_{2}$ symmetry whereas $\mathrm{Pd}_{13}$ is found to be icosahedral fragment $\left(\mathrm{C}_{2}\right)$. These resemble the groundstate structures of the same sizes investigated previously $[14,66]$. The predicted $\mathrm{Pd}_{11}$ structure is energetically preferred over the next lowest-lying structure by only $2 \mathrm{meV}$. The competitive isomer for $\mathrm{Pd}_{14}$ also has a distorted hexagonal bilayer (HBL) structure, but with lower symmetry $\left(\mathrm{C}_{1}\right)$ and a relative energy of $0.09 \mathrm{eV}$. The global minimum of $\mathrm{Pd}_{13}$ reported here is found to be different from the compact icosahedron structure predicted as the GM in references [67-69].

The GM for $\mathrm{Pd}_{12}$ has a $\left(\mathrm{C}_{1}\right)$ buckled mono-planar (BMP) structure, which is similar to that observed recently by Xing and co-workers [32]. The GM of $\mathrm{Pd}_{15}$ is a buckled biplanar (BBP) structure. In the case of $\mathrm{Pd}_{N}$ clusters, $N=16-18$ atom, the global minima are found to be pseudo-spherical filled cage structures and agree with the fcc-like growth pathway observed previously for 16-20 atoms $[14,32,70]$.

\subsubsection{Au-Pd clusters}

The global minima for all compositions of $\mathrm{Au}_{m} \mathrm{Pd}_{n}$ clusters, $11 \leq m+n \leq 18$, are shown in Figures $5-8$. Tables S2-S5 list the energies, coordinates and point groups (see the Supporting Information).

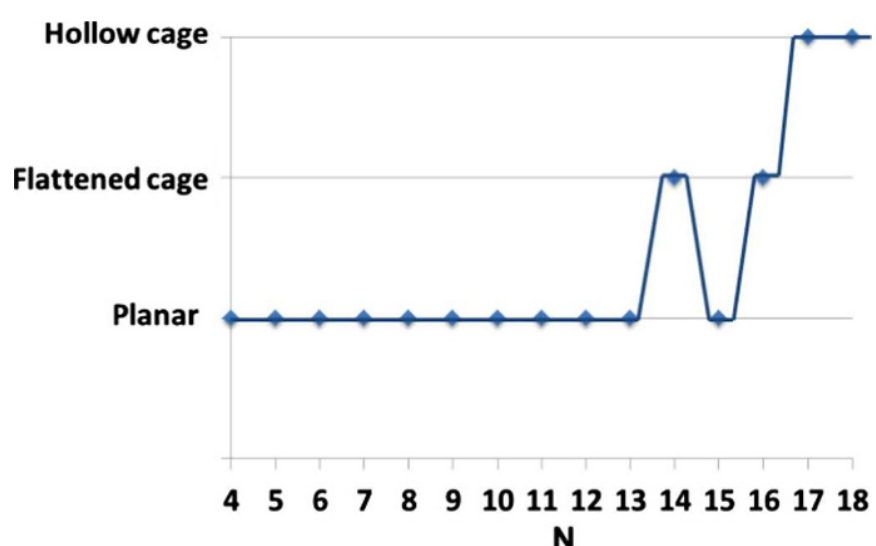

Fig. 2. Evolution of structural motifs for Au clusters. The structural properties for sizes $N=4-10$ are taken from reference [37].
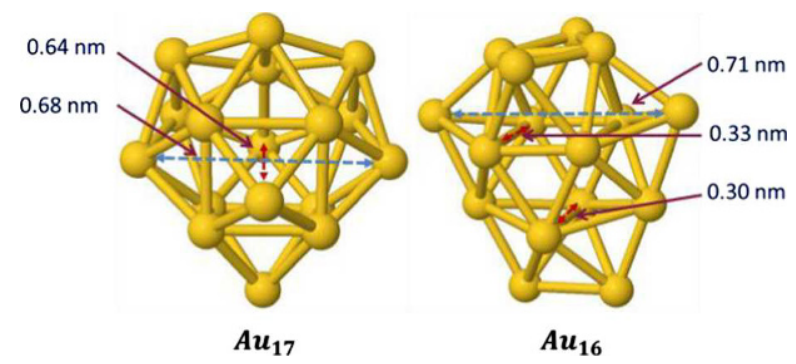

Fig. 3. The shortest-axis lengths of the predicted hollow cage structure of $\mathrm{Au}_{17}$ and flattened cage structure of $\mathrm{Au}_{16}$.

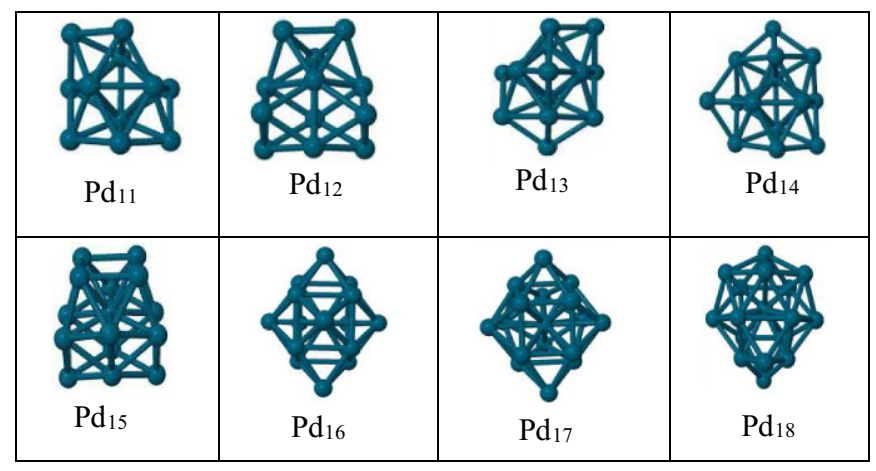

Fig. 4. Putative global minimum structures for $\operatorname{Pd}_{N}$ clusters, $N=11-18$.

As shown in Figure 5, all the predicted structures of mono-gold-doped Pd clusters $\left(\mathrm{Au}_{1} \mathrm{Pd}_{n}, n=N-1=10\right.$ 17) are 3D. For $N=11,12$ and 18, replacement of a single $\mathrm{Pd}$ atom in $\mathrm{Pd}_{N}$ by an $\mathrm{Au}$ atom yields geometries which are significantly distorted from the pure clusters. For sizes $N=13,15$ and 16, the Au-doped Pd clusters are similar to their pure $\mathrm{Pd}$ species whereas for $N=14$ and 17 atoms, the $\mathrm{Au}$-doped structures are quite different from the pure Pd clusters.

$\mathrm{Au}_{1} \mathrm{Pd}_{n} n=10-13$ show icosahedral (Ih) derivatives; as for $\mathrm{Au}_{1} \mathrm{Pd}_{10}, \mathrm{Au}_{1} \mathrm{Pd}_{11}$, and $\mathrm{Au}_{1} \mathrm{Pd}_{12}$ clusters, the most stable structure is an Ih fragment, while $\mathrm{Au}_{1} \mathrm{Pd}_{13}$ is an $\mathrm{Ih}$ fragment which can be considered as an incomplete $\mathrm{M}_{19}$ 


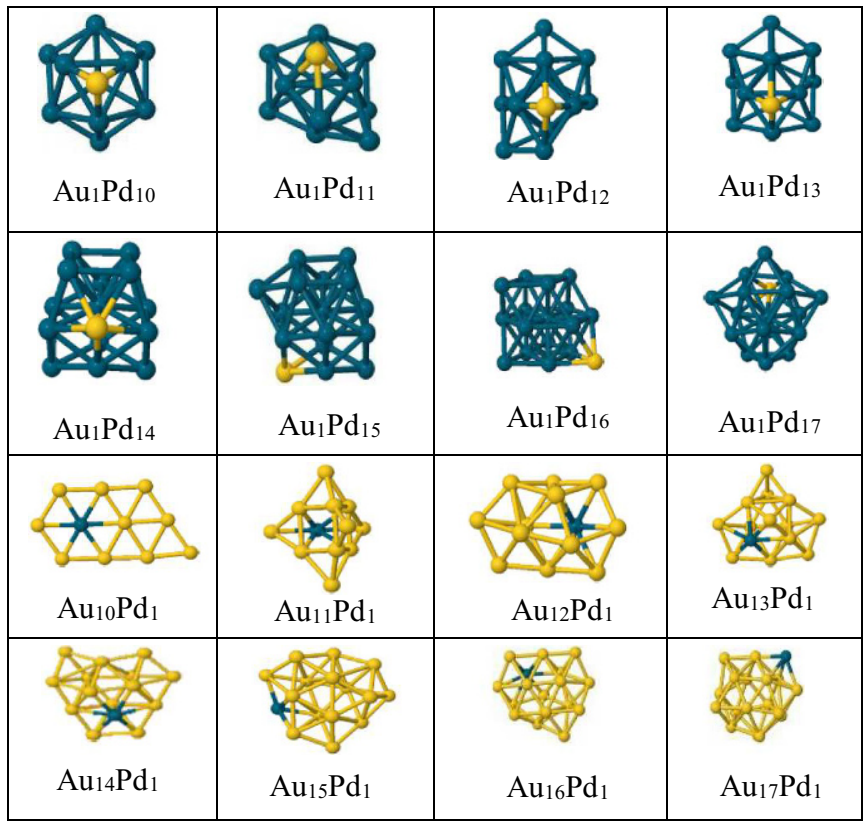

Fig. 5. Putative global minimum structures for mono-doped clusters, $N=11-18$. Au and $\mathrm{Pd}$ atoms are shown (here, and in subsequent figures) in yellow and blue, respectively.

double icosahedron. $\mathrm{Au}_{1} \mathrm{Pd}_{14}$ is found to be similar to its pure Pd counterpart $\left(\mathrm{Pd}_{15}\right)$ by showing a buckled biplanar (BBP) structure. GM of $\mathrm{Au}_{1} \mathrm{Pd}_{15}$ is found to be pseudospherical filled cage structure and can be considered as a bicapped truncated decahedron (tDh). For $\mathrm{Au}_{1} \mathrm{Pd}_{16}$, the GM is a hcp-based structure. GM of $\mathrm{Au}_{1} \mathrm{Pd}_{17}$ is a filled cage-like structure. In all cases of Au-doped Pd clusters, the unique $\mathrm{Au}$ is located in a low-connectivity surface site, in particular in capping sites on the $\mathrm{Pd}$ polyhedral cluster.

Figure 5 shows that all GM of $\mathrm{Au}_{m} \mathrm{Pd}_{1} m=10-17$ clusters are $3 \mathrm{D}$, except for planar $\mathrm{Au}_{10} \mathrm{Pd}_{1}$. Substitution of one $\mathrm{Au}$ atom by $\mathrm{Pd}$ in pure $\mathrm{Au}$ clusters is responsible for the shift of the 2D-3D transition point from 14and 16 -atom clusters to 12 atoms $\left(\mathrm{Au}_{11} \mathrm{Pd}_{1}\right)$. This is in disagreement with the $2 \mathrm{D}-3 \mathrm{D}$ transition point suggested by Zanti and Peeters at size $N=8$ [35]. However, in continuation with the planar structural pattern observed for $N=4-10 \mathrm{Pd}$-doped Au clusters previously [37], the gas-phase $\mathrm{Au}_{10} \mathrm{Pd}_{1}$ global minimum is also planar. A noncompact 3D structure is the GM for $\mathrm{Au}_{11} \mathrm{Pd}_{1}$. The other mono-substituted clusters, $\mathrm{Au}_{12} \mathrm{Pd}_{1}-\mathrm{Au}_{17} \mathrm{Pd}_{1}$ have flattened cage structures. $\mathrm{Au}_{17} \mathrm{Pd}_{1}$, for example, shows more condensed cage compared with a pseudo-spherical hollow cage structure of pure $\mathrm{Au}_{18}$. The $\mathrm{Pd}$ atoms are located in or close to the centre of the clusters in high-coordination sites. The deviation from planarity generally occurs near this $\mathrm{Pd}$ centre, as previously reported for $N \leq 14$ clusters [35].

The putative GM for greater degrees of substitution are shown in Figures $6(N=11-14), 7(N=15-17)$ and 8 $(N=18)$.

For $N=11$, doping two $\mathrm{Au}$ atoms into $\mathrm{Pd}_{11}$ clusters yields bilayer-structure, with the $\mathrm{Au}$ atoms in capping positions. All GM from $\mathrm{Au}_{3} \mathrm{Pd}_{8}$ to $\mathrm{Au}_{5} \mathrm{Pd}_{6}$ clusters have 3-layer hexagonal close-packed (hcp) structures, while $\mathrm{Au}_{6} \mathrm{Pd}_{5}-\mathrm{Au}_{8} \mathrm{Pd}_{3}$ have symmetrical or distorted Ih fragment structures which are similar to the GM found for $\mathrm{Au}_{1} \mathrm{Pd}_{10}$. In contrast, $\mathrm{Au}_{9} \mathrm{Pd}_{2}$ is found to be similar in shape to pure $\mathrm{Pd}_{11}$, showing a hexagonal bilayer (HBL) fragment.

For $N=12$, doping up to ten $\mathrm{Au}$ atoms changes the structure of gas-phase $\mathrm{Au}_{1} \mathrm{Pd}_{11}$ and $\mathrm{Au}_{2} \mathrm{Pd}_{10}$ from an $\mathrm{Ih}$ fragment to fcc-like motifs. They are 3-layer structures up to $\mathrm{Au}_{7} \mathrm{Pd}_{5}$, but they are incomplete for $\mathrm{Au}_{8} \mathrm{Pd}_{3}$ $\mathrm{Au}_{10} \mathrm{Pd}_{2}$. The structural configurations observed here for $\mathrm{Au}_{3} \mathrm{Pd}_{9}-\mathrm{Au}_{7} \mathrm{Pd}_{5}$ agree with the clusters reported previously by Zanti and Peeters [35], although their homotopic distributions are different.

For $N=13$, the icosahedral fragment observed for $\mathrm{Pd}_{13}$ is the global minimum for $\mathrm{Au}_{1} \mathrm{Pd}_{12}-\mathrm{Au}_{3} \mathrm{Pd}_{10}$ clusters. $\mathrm{Au}_{7} \mathrm{Pd}_{6}$ has a 3 -layer hcp structure. The interactions between $\mathrm{Au}$ and $\mathrm{Pd}$ atoms favours fcc-like motifs for $\mathrm{Au}_{4} \mathrm{Pd}_{9}-\mathrm{Au}_{6} \mathrm{Pd}_{7}$ and $\mathrm{Au}_{8} \mathrm{Pd}_{5}-\mathrm{Au}_{11} \mathrm{Pd}_{2}$ clusters, having octahedral-based configurations. These fcc-like motifs were observed previously [35] for $\mathrm{Au}_{4} \mathrm{Pd}_{9}, \mathrm{Au}_{6} \mathrm{Pd}_{7}$ and $\mathrm{Au}_{8} \mathrm{Pd}_{5}-\mathrm{Au}_{10} \mathrm{Pd}_{3}$ clusters, but with different homotopic distributions, apart from $\mathrm{Au}_{6} \mathrm{Pd}_{7}$.

For $N=14$, the gas-phase structure of $\mathrm{Au}_{1} \mathrm{Pd}_{13}$, $\mathrm{Au}_{3} \mathrm{Pd}_{11}, \mathrm{Au}_{5} \mathrm{Pd}_{9}$ and $\mathrm{Au}_{7} \mathrm{Pd}_{7}$ are all Ih-based fragments, whereas a distorted hexagonal bilayer (HBL) structure is the GM for $\mathrm{Au}_{2} \mathrm{Pd}_{12}$. For $\mathrm{Au}_{4} \mathrm{Pd}_{10}, \mathrm{Au}_{6} \mathrm{Pd}_{8}$ and $\mathrm{Au}_{10} \mathrm{Pd}_{4}$ clusters, the gas-phase global minima are fcc-fragments, and $\mathrm{Au}_{8} \mathrm{Pd}_{6}$ and $\mathrm{Au}_{9} \mathrm{Pd}_{5}$ show a continuation of the fcc structural growth, having complete octahedron motifs. These motifs have been reported previously [35] with different homotopic distributions. A clear structural transition from fcc-like structure to complex condensed cage structures occurs for $\mathrm{Au}_{11} \mathrm{Pd}_{3}-\mathrm{Au}_{13} \mathrm{Pd}_{1}$ clusters.

For $N=15$, the buckled biplanar (BBP) structure of $\mathrm{Pd}_{15}$ remains the GM when doped with up to two $\mathrm{Au}$ atoms. GM of $\mathrm{Au}_{3} \mathrm{Pd}_{12}$ is found to be hcp structure. Distorted hexagonal bilayer (HBL) structures are the lowest energy isomers when doping eight and nine atoms of $\mathrm{Au}$ whereas distorted fcc-like structures are found on doping 4-6 and 10-12 atoms of Au. Fused cage structures are the favoured structures for $\mathrm{Au}_{7} \mathrm{Pd}_{8}, \mathrm{Au}_{13} \mathrm{Pd}_{2}$ and $\mathrm{Au}_{14} \mathrm{Pd}_{1}$.

For $N=16$, although a bicapped truncated decahedron $\left(\mathrm{tDh}\right.$ ) is the favoured structure for $\mathrm{Pd}_{16}$ (and also $\mathrm{Au}_{1} \mathrm{Pd}_{15}$ ), doping up to five atoms of $\mathrm{Au}$ results in putative GM with hcp structures. $\mathrm{Au}_{6} \mathrm{Pd}_{10}, \mathrm{Au}_{7} \mathrm{Pd}_{9}$ and $\mathrm{Au}_{9} \mathrm{Pd}_{7}-\mathrm{Au}_{11} \mathrm{Pd}_{5}$ clusters show compact capped pentagonal prism and icosahedral structures, whereas $\mathrm{Au}_{12} \mathrm{Pd}_{4}$ is a hcp-based fragment. A pseudo-spherical-like cage structure is the global minimum for both $\mathrm{Au}_{8} \mathrm{Pd}_{8}$ and $\mathrm{Au}_{13} \mathrm{Pd}_{3}$ while $\mathrm{Au}_{14} \mathrm{Pd}_{2}$ has a bicapped 13-atom close packed layer and $\mathrm{Au}_{15} \mathrm{Pd}_{1}$ a flattened cage structure.

For $N=17$, doping two or three $\mathrm{Au}$ atoms retains the cage structure of $\mathrm{Pd}_{17}$, though the mono-doped $\mathrm{Pd}$ cluster has a different (hcp), structure. $\mathrm{Au}_{4} \mathrm{Pd}_{13}$ is an icosahedral fragment, which is related to the structure of $\mathrm{Au}_{10} \mathrm{Pd}_{7}$. Moving to $\mathrm{Au}_{6} \mathrm{Pd}_{11}$, the GM is a capped hcp structure, whereas $\mathrm{Au}_{5} \mathrm{Pd}_{12}$ has structure intermediate between those of the $\mathrm{Au}_{4} \mathrm{Pd}_{13}$ and $\mathrm{Au}_{6} \mathrm{Pd}_{11}$ clusters. The predicted structure for $\mathrm{Au}_{7} \mathrm{Pd}_{10}$ is a mixture of icosahedral and octahedral structures, while the GM for $\mathrm{Au}_{8} \mathrm{Pd}_{9}$ 
is a fused icosahedral structure. The GM for $\mathrm{Au}_{9} \mathrm{Pd}_{8}$ has an fcc structure, while hcp-based structures are found for $\mathrm{Au}_{11} \mathrm{Pd}_{6}-\mathrm{Au}_{13} \mathrm{Pd}_{4}$. Doping up to three $\mathrm{Pd}$ atoms into the $\mathrm{Au}_{17}$ cluster gives complex cage structures, though, unlike $\mathrm{Au}_{17}$, they are not hollow.

For $N=18$, as for $\mathrm{Pd}_{18}$, the predicted GM for $\mathrm{Au}_{2} \mathrm{Pd}_{16}$ is filled pseudo-spherical cage, whereas $\mathrm{Au}_{3} \mathrm{Pd}_{15}$ and $\mathrm{Au}_{4} \mathrm{Pd}_{14}$ are based on icosahedral fragments. The structures of $\mathrm{Au}_{5} \mathrm{Pd}_{13}-\mathrm{Au}_{7} \mathrm{Pd}_{11}$ can be described as polytetrahedral, composed of mainly fused pentagonal bipyramids with (in some cases) hexagonal bipyramids. Fused tricapped buckled biplanar (BBP) structure is the favoured structure for $\mathrm{Au}_{10} \mathrm{Pd}_{8}$. Filled pseudo-spherical structures are the putative GM for $\mathrm{Au}_{8} \mathrm{Pd}_{10}, \mathrm{Au}_{9} \mathrm{Pd}_{9}$ and $\mathrm{Au}_{16} \mathrm{Pd}_{2}$ clusters. Fusing of hexagonal bipyramid structures generates a shell-like structure for the $\mathrm{Au}_{11} \mathrm{Pd}_{7}$ cluster. A filled spherical-like structure is also the global minima for the $\mathrm{Au}_{12} \mathrm{Pd}_{6}$ and $\mathrm{Au}_{13} \mathrm{Pd}_{5}$ clusters which are formed by fusing of hexagonal bipyramid, pentagonal bipyramid and icosahedral structures. Fusing of icosahedral fragments makes a spherical-like cage structure for the $\mathrm{Au}_{14} \mathrm{Pd}_{4}$ cluster, whereas fusing of hexagonal bipyramids and pentagonal bipyramids yields the crown-like structure for $\mathrm{Au}_{15} \mathrm{Pd}_{3}$. Finally, a capped flattened cage structure is the global minima for $\mathrm{Au}_{17} \mathrm{Pd}_{1}$, although the pure $\mathrm{Au}_{18}$ cluster is a hollow cage.

\subsection{Stability}

Generally, the observed preference of $\mathrm{Au}$ atoms for low coordination surface positions (especially capping sites) and Pd atoms for high coordination core and central sites can be explained with reference to bulk cohesive and surface energies which are higher for Pd: average low-index surface energies $\left(E_{\text {sur }}\right)$ and cohesive energy $\left(E_{c o h}\right)$ are $96.8 \mathrm{meV} \AA^{-2}$ and $3.81 \mathrm{eV} \mathrm{atm}^{-1}$, respectively, for $\mathrm{Au}$ and $131 \mathrm{meV}^{-2}$ and $3.89 \mathrm{eV} \mathrm{atm}^{-1}$, respectively, for Pd. The stronger $\mathrm{Pd}-\mathrm{Pd}$ bonds and lower Au surface energy favours Pd-rich cores and Au-rich outer shells (though this depends on the cluster size and composition). This preference is reinforced by the sizes of the atoms, as the atomic radius of $\mathrm{Pd}$ is smaller than for $\mathrm{Au}$ by $0.06 \AA$, so the larger $\mathrm{Au}$ atoms are more easily accommodated in low coordination sites. The small difference in electronegativity values of $\operatorname{Pd}(2.2)$ and $\mathrm{Au}(2.4)$ can lead to weak $(\mathrm{Pd}$ to $\mathrm{Au}) \mathrm{s}$ electron transfer [34,38] (favouring mixing in larger clusters), but favouring surface $\mathrm{Au}$ in smaller clusters, as the more electronegative $\mathrm{Au}$ atom can better stabilise the negative charge that tends to build up on the cluster surface [71]. However, it should be noted that for larger clusters reverse (Au to $\mathrm{Pd}$ ) $d$-band electron donation can also occur [72,73].

The stabilities of the clusters, and their structural preferences, can be investigated by calculating the excess energy $\Delta$, binding energy $E_{b}$, and second difference in energy $\Delta_{2} E$, which are defined in equations (1)-(3). Tables S6-S9 list the values of these energies for all clusters studied here (see the Supporting Information).

The relative stabilities of clusters of different nuclearities can be studied by calculating the second difference

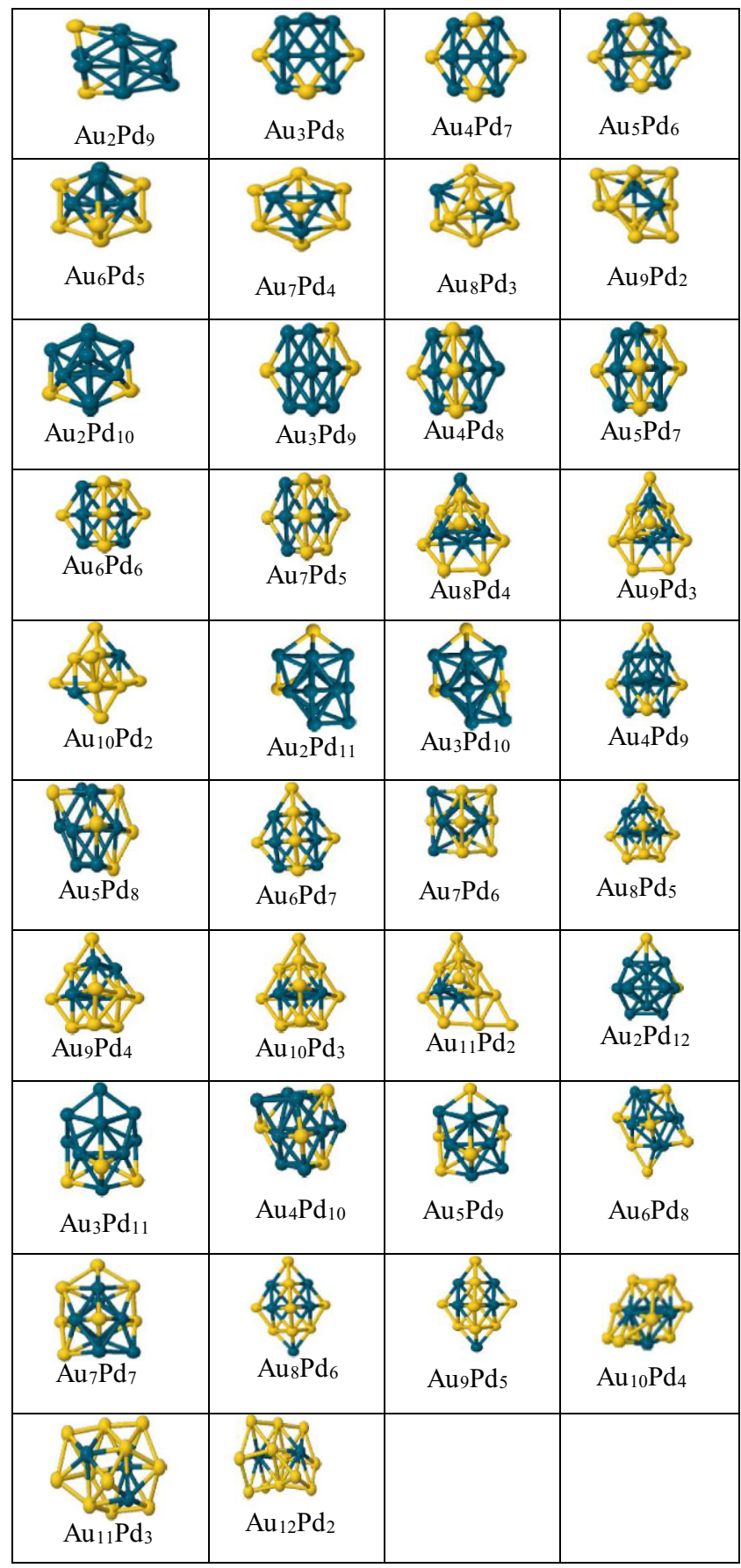

Fig. 6. Putative global minimum structures for all compositions (excluding mono-doping) of $\mathrm{AuPd}$ nanoalloys with sizes $N=11-14$.

in energy $\Delta_{2} E$, which indicates the stability of an $\mathrm{N}$ atom cluster with respect to neighbouring sizes. Figure 9 shows a plot of $\Delta_{2} E$ for $\mathrm{Au}$ and $\mathrm{Pd}$ clusters and their mono-substituted clusters as a function of cluster size. The relatively stable clusters are indicated by significant positive peaks.

Compared to their neighbours, even-numbered clusters $\mathrm{Au}_{12}$ (2D), $\mathrm{Au}_{14}$ (3D) and $\mathrm{Au}_{16}$ (3D) have high relative 


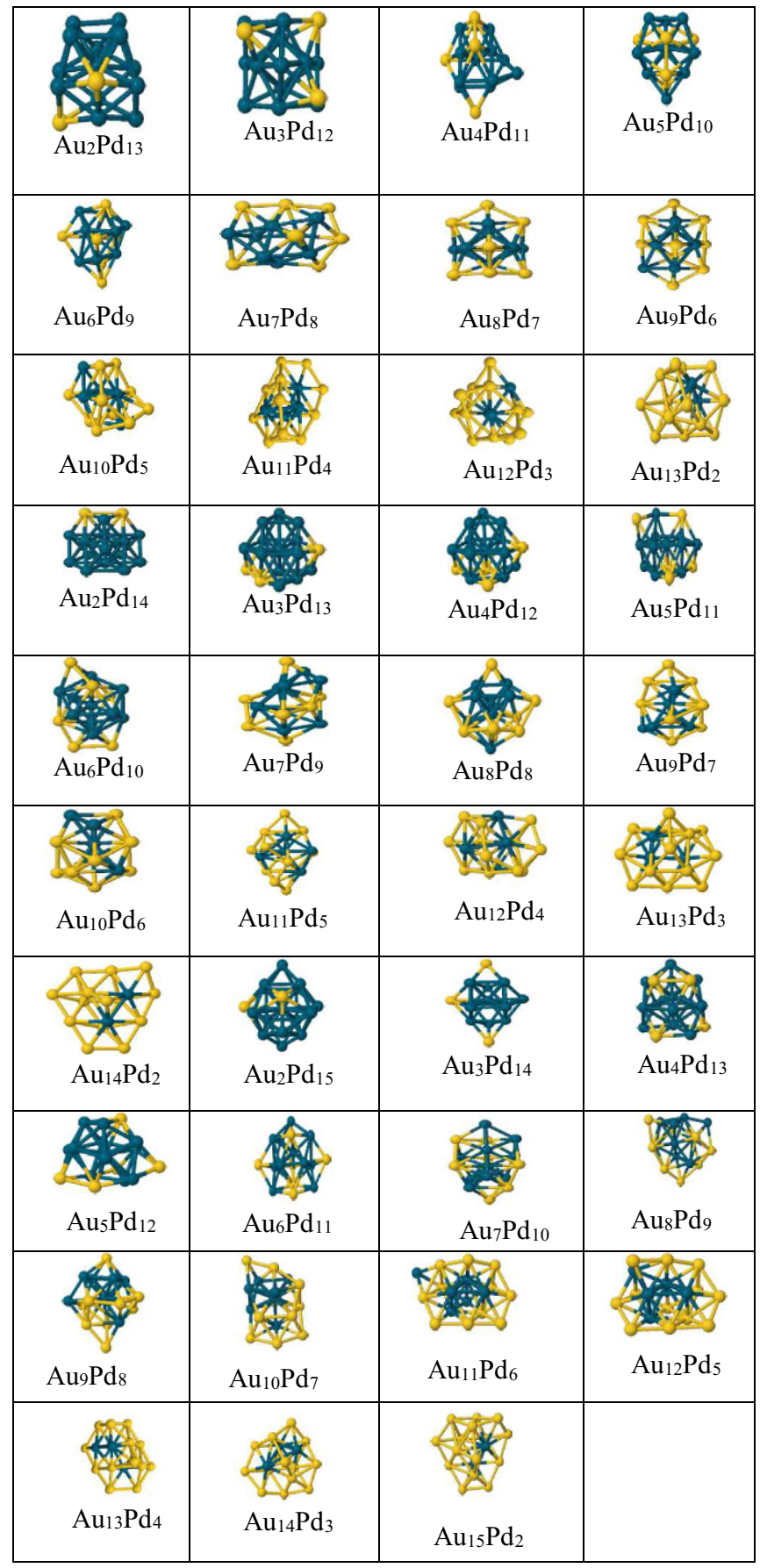

Fig. 7. Putative global minimum structures for all compositions (excluding mono-doping) of AuPd nanoalloys with sizes $N=15-17$.

stabilities, corresponding to peaks in $\Delta_{2} E$, with oddnumbered clusters being relatively unstable (troughs in $\Delta_{2} E$ ). This even-odd behaviour is due to the fact that the $\mathrm{Au}$ atom has an unpaired $s$ electron, and so reflects the greater stability of Au clusters (either 2D or 3D) with an even number of electrons, over those with an odd number [74-77]. The most stable cluster is $2 \mathrm{D} \mathrm{Au}_{12}$, although its $\Delta_{2} E$ energy is lower (by $0.67 \mathrm{eV}$ ) than the magic size $\mathrm{Au}_{6}$ observed recently [37]. Doping a single $\mathrm{Pd}$ atom into Au clusters shifts the stability from the even-number

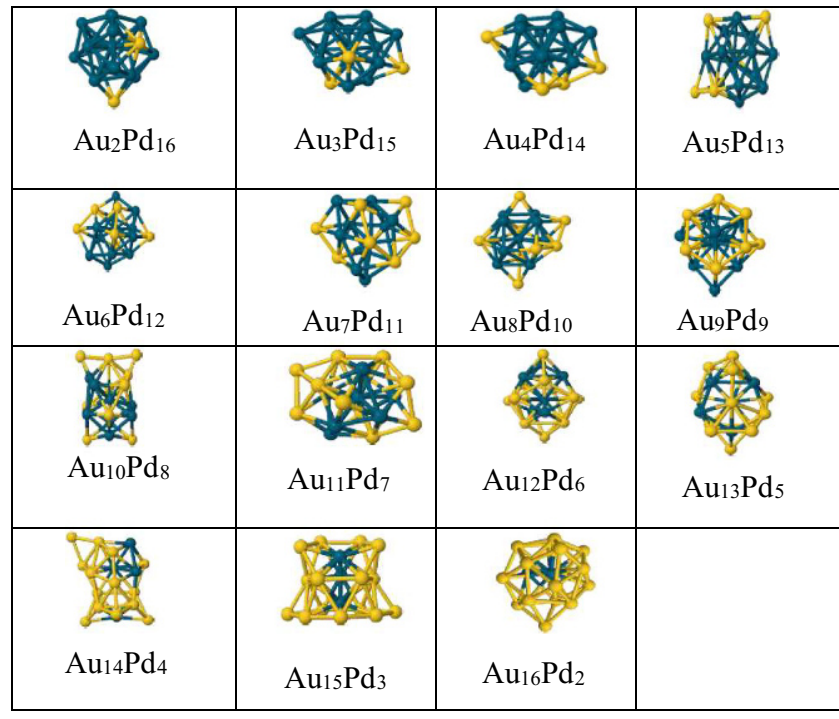

Fig. 8. Putative global minimum structures for all compositions (excluding mono-doping) of AuPd nanoalloys with size $N=18$.
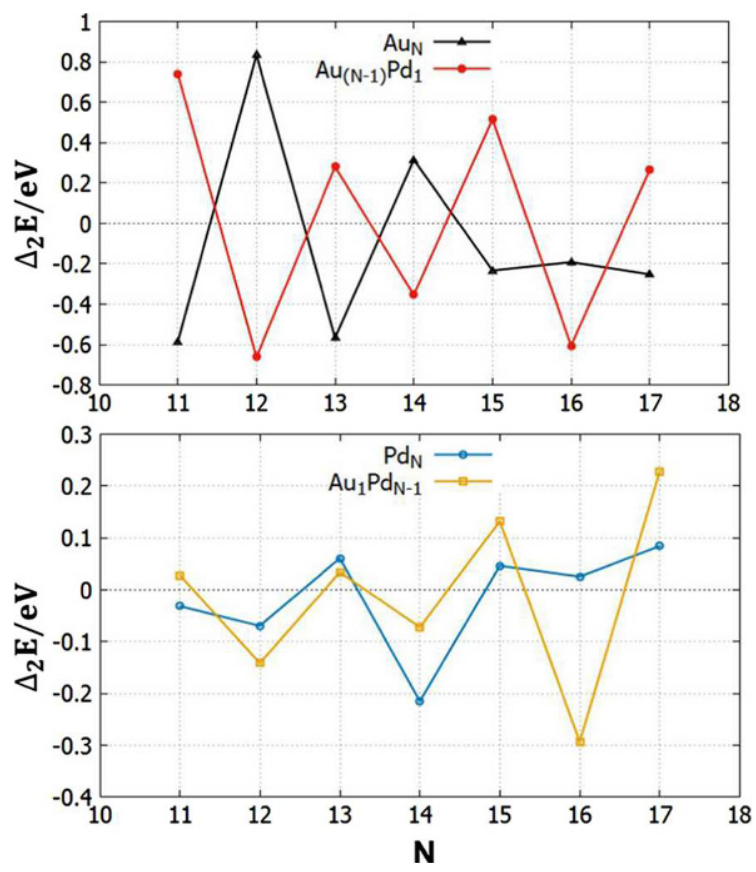

Fig. 9. Second difference in energy $\left(\Delta_{2} E\right)$ of pure $A u$ and $\mathrm{Pd}$-doped Au clusters (top); and pure Pd and Au-doped Pd clusters (bottom) with respect to the total number of atoms $(\mathrm{N})$.

$(N=12,14$, and 16) clusters to odd-number $(N=11$, 13,15 , and 17) clusters. This shift occurs because an oddelectron $\mathrm{Au}$ atom is replaced by an even-electron $\mathrm{Pd}$ atom, so clusters with odd values of $N$ (e.g. $\mathrm{Au}_{10} \mathrm{Pd}_{1}$, which has the highest $\Delta_{2} E$ value) now have an even number of electrons, and consequently are stable with respect to neighbouring cluster sizes.

As the Pd atom has an even number of electrons, there is no enhanced electronic stability for even $N$. In fact, odd numbered $\mathrm{Pd}_{N}$ clusters are relatively more stable than for 

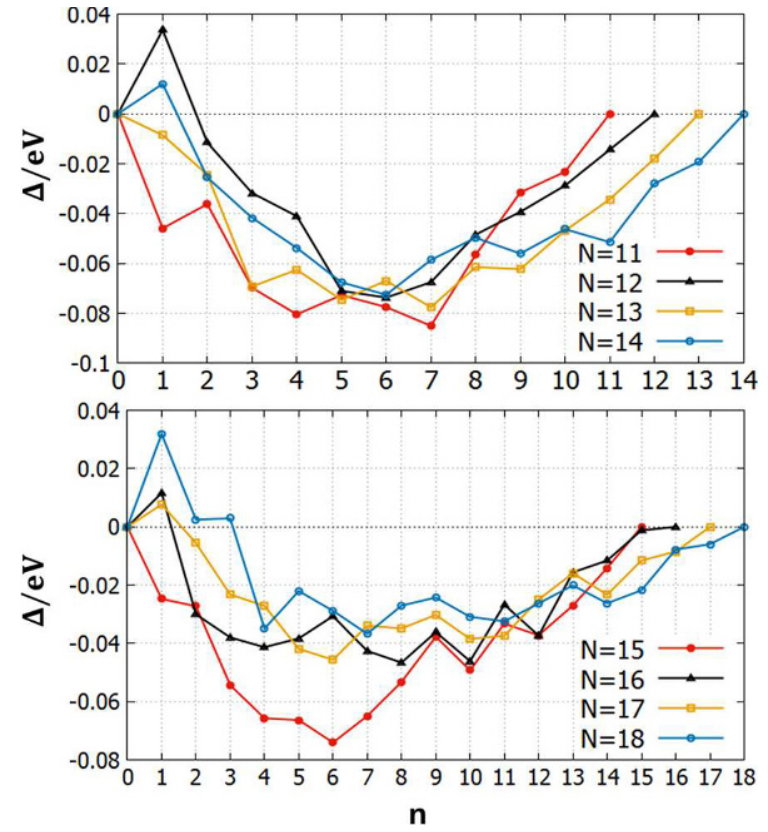

Fig. 10. Excess energy $(\Delta)$ as a function of the number of $\mathrm{Pd}$ atoms $(n)$ for all compositions of $11 \leq N \leq 18$ for $\mathrm{Au}_{m} \mathrm{Pd}_{n}$ clusters, where $N=m+n$.

even $N$, which could be attributed to packing considerations. This behaviour is consistent with the significant effect of $\mathrm{Pd}$ atoms in weakening the odd-even fluctuation of stability of $\mathrm{Ag}$ and $\mathrm{Cu}$ clusters reported recently by Kahnouji et al. [78] Doping a single $\mathrm{Au}$ atom into $\mathrm{Pd}_{N}$ clusters generates odd-electron clusters for all values of $N$. The shape of the $\Delta_{2} E$ plot does not change and there is no shift in the odd-even pattern. Interestingly, for the larger sizes $(N=15-17)$, the extremes in $\Delta_{2} E$ are greater for the Au-doped clusters than for the corresponding pure $\operatorname{Pd}_{N}$ clusters. The size $N=17$ shows the most relatively stable structure for both pure $\mathrm{Pd}$ and $\mathrm{Au}$-doped Pd clusters, although $\mathrm{Pd}_{6}$ has been predicted to have a $\Delta_{2} E$ value $0.34 \mathrm{eV}$ higher than the $\mathrm{Pd}_{17}$ cluster [37].

The effect of mixing $\mathrm{Au}$ with $\mathrm{Pd}$ in medium-sized clusters is studied by calculating the excess energy, $\Delta$. Excess energies as a function of the number of $\mathrm{Pd}$ atoms are shown in Figures 10 and 11 for all compositions of $11 \leq m+n \leq 18$ for $\mathrm{Au}_{m} \mathrm{Pd}_{n}$ clusters. Negative values of excess energies represent favourable mixing, whereas de-mixing is indicated by positive values of $\Delta$.

For $(N=m+n=11)$ clusters, the best mixing (most negative $\Delta$ ) is found at $\mathrm{Au}_{4} \mathrm{Pd}_{7}$, whereas $\mathrm{Au}_{1} \mathrm{Pd}_{10}$ exhibits a weak mixing behaviour. All $(m+n=$ 12) nanoalloys show a strong mixing tendency except $\mathrm{Au}_{11} \mathrm{Pd}_{1}$ and the strongest mixing is for $50 \% \mathrm{Pd}$ composition. For $(m+n=13), \mathrm{Au}_{6} \mathrm{Pd}_{7}$ shows the most energetically favourable mixing. For $(m+n=14)$, only $\mathrm{Au}_{13} \mathrm{Pd}_{1}$ shows a strong de-mixing tendency and the most favourable mixing is for $\mathrm{Au}_{8} \mathrm{Pd}_{6}$. All $(m+n=15)$ clusters favour mixing, with the strongest mixing for $\mathrm{Au}_{9} \mathrm{Pd}_{6}$. For $(m+n=16,17$ and 18), all Pd-doped Au clusters are energetically unfavourable compared to pure Pd and $\mathrm{Au}$ clusters, while the reverse is true for all other mixed clusters. The strongest mixing tendency at these sizes is observed for $\mathrm{Au}_{8} \mathrm{Pd}_{8}, \mathrm{Au}_{11} \mathrm{Pd}_{6}$ and $\mathrm{Au}_{11} \mathrm{Pd}_{7}$, though the magnitude of $\Delta$ is much smaller than for $m+n=15$ $\left(\mathrm{Au}_{9} \mathrm{Pd}_{6}\right)$. In fact, we find that the optimum values of $\Delta$ for $N=11-15$ (approx. -0.075 to $-0.085 \mathrm{eV}$ ) are quite similar but have approximately twice the magnitude of the larger clusters $(N=16-18)$.

The stabilities of nanoclusters relative to their constituent ground state atoms are obtained by calculating the binding energy per atom, $E_{b}$. According to the plots of $E_{b}$ shown in Figures 12 and 13, the binding energy generally increases with increasing cluster size (though local switches in order along the $y$-axis correspond to peaks or troughs in the excess energy plots), as previously reported $[35,37]$. It can also be seen that the binding energy increases with increasing $\mathrm{Pd}$ composition (reflecting the stronger $\mathrm{Pd}-\mathrm{Pd}$ and $\mathrm{Pd}-\mathrm{Au}$ bonds compared to $\mathrm{Au}-\mathrm{Au}$ ), although the mono-Pd-doped $\mathrm{Au}$ clusters have lower $E_{b}$ values than pure $\mathrm{Au}$ clusters for even sizes ( $N=12,14,16$, and 18 atoms). This is consistent with the shift in excess energies upon mono-doping of Pd into $\mathrm{Au}$ clusters seen in Figure 10. However, this disagrees with the relative stability observed for mixed clusters compared with their pure clusters for small sized $\mathrm{Au}-\mathrm{Pd}$ clusters $N=4-10$ except for $N=6$, which is found to be a magic for pure $\mathrm{Au}$ and $\mathrm{Pd}$ clusters [37].

HOMO-LUMO gaps could indicate the structural stabilities of the clusters. High stability can be indicated by high HOMO-LUMO gaps. Figure S1 (see the Supporting Information) shows HOMO-LUMO gap versus number of $\mathrm{Pd}$ atoms for all clusters. For Au clusters, in line with $\Delta_{2} E$ results, the even-numbered clusters $N=12,14,16$, and 18 atoms have high stabilities whereas the odd-numbered clusters are relatively unstable. HOMO-LUMO gaps of Pd clusters are range between $(0.06-0.15 \mathrm{eV})$. For nanoalloys, higher HOMO-LUMO gaps correlate well with the suggested high stability (high mixing energy $\Delta$ ) compositions for $N=12,14,16$, and 17. However, HOMO-LUMO gaps disagree with the stability found for $N=13$ and 18 . For $N=11$, the high HOMO-LUMO gaps observed for $\mathrm{Au}_{7} \mathrm{Pd}_{4}, \mathrm{Au}_{8} \mathrm{Pd}_{3}$ and $\mathrm{Au}_{10} \mathrm{Pd}_{1}$ disagree with $\Delta$ results except $\mathrm{Au}_{7} \mathrm{Pd}_{4}$ which is found to be stable in both. For $N=15, \mathrm{Au}_{11} \mathrm{Pd}_{4}$ is found to be stable according to $\Delta$ and HOMO-LUMO gap whereas the results do not match for $\mathrm{Au}_{14} \mathrm{Pd}_{1}$.

The possible permutations of two different metals $(\mathrm{Au}$ and $\mathrm{Pd}$ ) in the system can be investigated by the systematic homotop search. The number of homotops grows exponentially with the size of the cluster [38]. Hence many symmetry inequivalent homotops may have been missed by our BPGA-DFT search. In this context, we have studied the structural energies of proposed homotops of the mono-substituted clusters which are built based on their GM and minimized at the DFT level using the VASP code. Figures S2-S9 (see the Supporting Information) show the relative energies $\Delta E$ against symmetry inequivalent homotops for all mono-substituted clusters. For our system, the BPGA-DFT search for all mono-substituted 


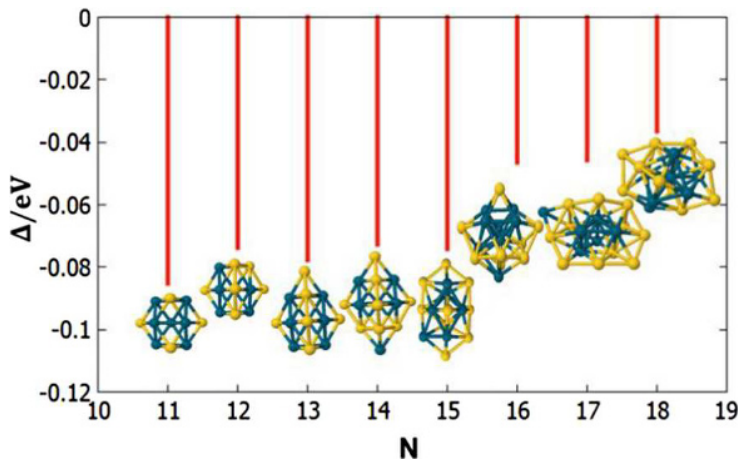

Fig. 11. Plot of the lowest excess energy $(\Delta)$, and the corresponding compositional isomer, calculated for each cluster size $(N=11-18)$ for $\mathrm{Au}_{m} \mathrm{Pd}_{n}$ clusters.

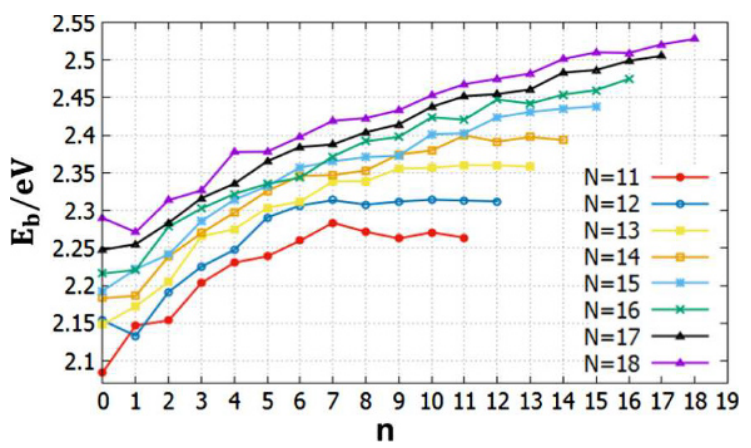

Fig. 12. Plot of binding energies of $\mathrm{Au}_{(N-n)} \mathrm{Pd}_{n}$ clusters for each cluster size $N=11-18$ against the number of $\mathrm{Pd}$ atoms $(n)$.

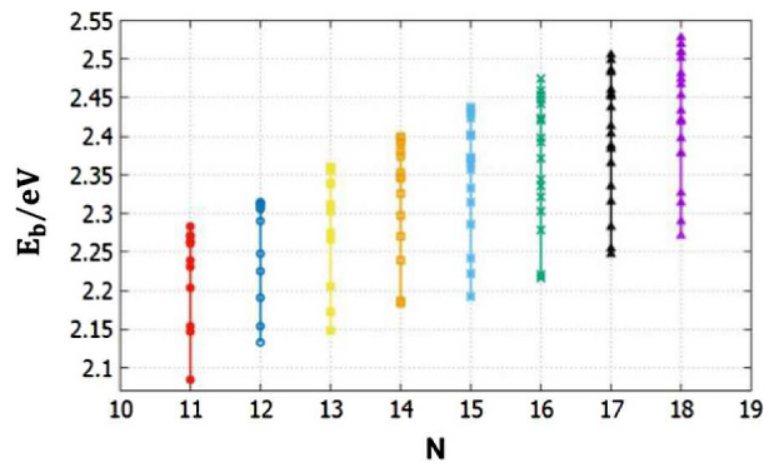

Fig. 13. The range of binding energies $\left(E_{b}\right)$, for all compositions of $\mathrm{Au}_{(N-n)} \mathrm{Pd}_{n}$ clusters, plotted against the total number of atoms $(N)$.

clusters $\mathrm{Au}_{1} \mathrm{Pd}_{n}$ and $\mathrm{Au}_{m} \mathrm{Pd}_{1}$ with 11 to 18 atoms, successfully found the lowest energy homotop as the global minimum.

\section{Conclusions}

We have applied the DFT based-Birmingham Parallel Genetic Algorithm (BPGA-DFT) to $\mathrm{Au}-\mathrm{Pd}$ nanoalloys ranging from 11 to 18 atoms and compared them with their pure clusters in the same size range. The BPGADFT approach has successfully found the global minima of the studied clusters. A GM structures were reinforced by the systematic homotop search for singly doped clusters.

2D close-packed planar layer, condensed flattened cage, and pseudo-spherical hollow cage structure are identified to be the three generic structure types for pure $\mathrm{Au}$ clusters. Although all Pd clusters obtained here are 3D, their structural motifs are found to be size-dependent; as a structural transition occurs at $N=15$ atoms from bilayer structures to filled cage structures. The structural behaviour for nanoalloys is controlled by the composition and size. Au atoms tend to be located in low-connectivity surface sites, in particular in capping sites, whereas Pd atoms generally prefer high-coordination positions in or close to the centre of the cluster.

The even-numbered Au clusters, $\mathrm{Au}_{12}$ (2D), $\mathrm{Au}_{14}$ (3D) and $\mathrm{Au}_{16}$ (3D) show high relative stability, corresponding to peaks in $\Delta_{2} E$. There is no enhanced electronic stability for even-numbered Pd clusters. A strong tendency of $\mathrm{Au}-$ Pd clusters to alloy was predicted from calculated mixing energies, with the exception of some mono-Pd-doped $\mathrm{Au}$ clusters. The stabilities of cluster relative to their constituent increase with increasing cluster size and are found to be higher for Pd and Pd-rich clusters.

\section{Supplementary Material}

The Supporting Information includes energies, structures and point group symmetries (Tables S1-S5); Excess Energies $\Delta$, Binding Energies $E_{b}$, and the second difference in energy $\Delta_{2} E$ (Tables S6-S9); the HOMO-LUMO gaps for all clusters (Fig. S1); and the relative energies $\Delta E$ for all symmetry inequivalent homotops of mono-substituted clusters (Figs. S2-S9).

H.A. Hussein is grateful to the University of Kufa and the Ministry of Higher Education and Scientific Research (Iraq) for the award of a PhD scholarship. Calculations were performed on the University of Birmingham's Blue-BEAR high-performance computer and ARCHER facilities, the UK National Supercomputing Service (http://www.archer.ac.uk) via membership of the UK's HPC Materials Chemistry Consortium, which is funded by EPSRC (EP/L000202), and "TOUCAN: Towards an Understanding of Catalysis on Nanoalloys" membership, which is funded by EPSRC under Critical Mass Grant EP/J010804/1. H.A. Hussein thanks Dr. Jack Davis for helpful advice and John Hey for valuable help.

\section{Author contribution statement}

R.L. Johnston designed the research project and supervised the work and H.A. Hussein carried out the calculations. All authors contributed to data interpretation and discussion of the results.

Open Access This is an open access article distributed under the terms of the Creative Commons Attribution License (http://creativecommons.org/licenses/by/4.0), which 
permits unrestricted use, distribution, and reproduction in any medium, provided the original work is properly cited.

\section{References}

1. B. Hammer, J.K. Norskov, Nature 376, 238 (1995)

2. F. Yang, D. Deng, X. Pan, Q. Fu, X. Bao, Natl. Sci. Rev. 2, 183 (2015)

3. J.K. Edwards, B. Solsona, E. Ntainjua, A.F. Carley, A.A. Herzing, C.J. Kiely, G.J. Hutchings, Science 323, 1037 (2009)

4. D.I. Enache, J.K. Edwards, P. Landon, B. Solsona-Espriu, A.F. Carley, A.A. Herzing, M. Watanabe, C.J. Kiely, D.W. Knight, G.J. Hutchings, Science 311, 362 (2006)

5. X. Liu, M. Conte, M. Sankar, Q. He, D.M. Murphy, D. Morgan, R.L. Jenkins, D. Knight, K. Whiston, C.J. Kiely, G.J. Hutchings, Appl. Catal. A: Gen. 504, 373 (2015)

6. I. Demiroglu, Z.Y. Li, L. Piccolo, R.L. Johnston, Catal. Sci. Technol. 6, 6916 (2016)

7. L. Piccolo, Z.Y. Li, I. Demiroglu, F. Moyon, Z. Konuspayeva, G. Berhault, P. Afanasiev, W. Lefebvre, J. Yuan, R.L. Johnston, Sci. Rep. 6, 35226 (2016)

8. L. Wang, Y. Yamauchi, J. Am. Chem. Soc. 132, 13636 (2010)

9. Z. Peng, H. Yang, J. Am. Chem. Soc. 131, 7542 (2009)

10. V. Mazumder, S. Sun, J. Am. Chem. Soc. 131, 4588 (2009)

11. E. Antolini, Energy Environ. Sci. 2, 915 (2009)

12. M. Shao, J. Power Sources 196, 2433 (2011)

13. K. Zhang, Y. Xiang, X. Wu, L. Feng, W. He, J. Liu, W. Zhou S. Xie, Langmuir 25, 1162 (2009)

14. Y. Mu, Y. Han, J. Wang, J. Wan G. Wang, Phys. Rev. A 84, 053201 (2011)

15. X. Teng, Q. Wang, P. Liu, W. Han, A.I. Frenkel, W. Wen, N. Marinkovic, J.C. Hanson, J.A. Rodriguez, J. Am. Chem. Soc. 130, 1093 (2008)

16. G. Schon, U. Simon, Colloid Polym. Sci. 273, 202 (1995)

17. Y.W. Lee, M. Kim, Y. Kim, S.W. Kang, J. Lee, S.W. Han, J. Phys. Chem. C 114, 7689 (2010)

18. J.W. Hong, Y.W. Lee, M. Kim, S.W. Kang, S.W. Han, Chem. Commun. 47, 2553 (2011)

19. F. Wang, C. Li, L. Sun, H. Wu, T. Ming, J. Wang, J. C. Yu, C. Yan, J. Am. Chem. Soc. 133, 1106 (2011)

20. D. Chen, C. Li, H. Liu, F. Ye, J. Yang, Sci. Rep. 5, 11949 (2015)

21. C. Henry, C. Chapon, S. Giorgio, C. Goyhenex, in Chemisorption and Reactivity on Supported Clusters and Thin Films, 1st edn., edited by R.M. Lambert, P. Gianfranco (Springer, Netherlands, 1997), Vol. 331, p. 117

22. R. Ismail, R. Ferrando, R.L. Johnston, J. Phys. Chem. C 117, 293 (2013)

23. L.O. Paz-Borbón, R.L. Johnston, G. Barcaro, A. Fortunelli, Eur. Phys. J. D 52, 131 (2009)

24. A. Logsdail, L.O. Paz-Borbón, R.L. Johnston, J. Comput. Theor. Nanosci. 6, 1 (2009)

25. V. Kaydashev, P. Ferrari, C. Heard, E. Janssens, R.L. Johnston, P. Lievens, Part. Part. Syst. Charact. 33, 364 (2016)

26. S. Vajda, M.J. Pellin, J.P. Greeley, C.L. Marshall, L.A. Curtiss, G.A. Ballentine, J.W. Elam, S. Catillon-Mucherie, P.C. Redfern, F. Mehmood, P. Zapol, Nat. Mater. 8, 213 (2009)
27. A. Shayeghi, R.L. Johnston, R. Schäfer, Phys. Chem. Chem. Phys. 15, 19715 (2013)

28. P. Gruene, D.M. Rayner, B. Redlich, A.F.G. van der Meer, J.T. Lyon, G. Meijer, A. Fielicke, Science 321, 674 (2008)

29. A.P. Woodham, A. Fielicke, in Structure \&s Bonding: Gold Clusters, Colloids and Nano-Particles, edited by M. Mingos (2014), Vol. 161, pp. 243-278

30. G.A. Bishea, M.D. Morse, J. Chem. Phys. 95, 5646 (2001)

31. G.A. Bishea, M.D. Morse, J. Chem. Phys. 95, 8779 (1991)

32. X. Xing, A. Hermann, X. Kuang, M. Ju, C. Lu, Y. Jin, X. Xia, G. Maroulis, Sci. Rep. 6, 19656 (2016)

33. H. Huan, Y. Chen, T. Wang, X. Ye, J. Nanoparticle Res. 18, 349 (2016)

34. F. Pittaway, L.O. Paz-Borbón, R.L. Johnston, H. Arslan, R. Ferrando, C. Mottet, G. Barcaro, A. Fortunelli, J. Phys. Chem. C 113, 9141 (2009)

35. G. Zanti, D. Peeters, J. Phys. Chem. A 114, 10345 (2010)

36. D. Palagin, J. Doye, Phys. Chem. Chem. Phys. 17, 28010 (2015)

37. H.A. Hussein, B.A. Davis, R.L. Johnston, Phys. Chem. Chem. Phys. 18, 26133 (2016)

38. R. Ferrando, J. Jellinek, R.L. Johnston, Chem. Rev. 108, 845 (2008)

39. D. Zitoun, M. Respaud, M. Fromen, M.J. Casanove, P. Lecante, C. Amiens, B. Chaudret, Phys. Rev. Lett. 89, $037203(2002)$

40. H. Cantera-Lóopez, J.M. Montejano-Carrizales, F. Aguilera-Granja, J.L. Morán-López, Eur. Phys. J. D 57, $61(2010)$

41. J. Davis, A. Shayeghi, S.L. Horswell, R.L. Johnston, Nanoscale 7, 14032 (2015)

42. F. Buendía, J.B.A. Davis, J.A. Vargas, M.R. Beltrán, R.L. Johnston, Phys. Chem. Chem. Phys. 18, 22122 (2016)

43. I. Demiroglu, K. Yao, H.A. Hussein, R.L. Johnston, J. Phys. Chem. C 121, 10773 (2017)

44. J.B.A. Davis, S.L. Horswell, R.L. Johnston, J. Phys. Chem. C 120, 3759 (2016)

45. https://bitbucket.org/JBADavis/bpga/

46. R.L. Johnston, Dalton Trans. 4193 (2003)

47. A. Shayeghi, D. Götz, J.B.A. Davis, R. Schäfer, R.L. Johnston, Phys. Chem. Chem. Phys. 17, 2104 (2015)

48. B. Bandow, B. Hartke, J. Phys. Chem. A 110, 5809 (2006)

49. D. Deaven, K. Ho, Phys. Rev. Lett. 75, 288 (1995)

50. G. Kresse, J. Hafner, Phys. Rev. B 47, 558 (1993)

51. J. Perdew, K. Burke, Y. Wang, Phys. Rev. B 54, 16533 (1996)

52. G. Kresse, Phys. Rev. B 59, 1758 (1999)

53. M. Methfessel, A.T. Paxton, Phys. Rev. B 40, 3616 (1989)

54. J. Wang, G. Wang, J. Zhao, Phys. Rev. B 66, 035418 (2002)

55. Y. Dong, M. Springborg, Eur. Phys. J. D 43, 15(2007)

56. H. Häkkinen, B. Yoon, U. Landman, X. Li, H.J. Zhai, L.S. Wang, J. Phys. Chem. A 107, 6168 (2003)

57. B. Assadollahzadeh, P. Schwerdtfeger, J. Chem. Phys. 131, 064306 (2009)

58. J. David, D. Guerra, A. Restrepo, Chem. Phys. Lett. 539540, 64 (2012)

59. V.B. Koutecky, J. Burda, R. Mitrić, M.G. Zampella, J. Chem. Phys. 117, 3120 (2002)

60. Y.C. Choi, W.Y. Kim, H.M. Lee, K.S. Kim, J. Chem. Theor. Comput. 5, 1216 (2009)

61. D.A. Gotz, R. Schafer, P. Schwerdtfeger, J. Comput. Chem. 34, 1975 (2013) 
62. M.P. Johansson, I. Warnke, A. Le, F. Furche, J. Phys. Chem. C 118, 29370 (2014)

63. W. Fa, C. Luo, J. Dong, Phys. Rev. B 72, 205428 (2005)

64. S. Bulusu, X.C. Zeng, J. Chem. Phys. 125, 154303 (2006)

65. S. Bulusu, X. Li, L. Wang, X.C. Zeng, PNAS 103, 8326 (2006)

66. M. J. Piotrowski, P. Piquini, J.L.F. Da Silva, Phys. Rev. B 81, 155446 (2010)

67. Y. Sun, M. Zhang, Phys. Rev. A 79, 043202 (2009)

68. J. Rogan, G. García, J.A. Valdivia, W. Orellana, A.H. Romero, R. Ramírez, M. Kiwi, Phys. Rev. B 72, 115421 (2005)

69. A. Vega, J. Rogan, W. Orellana, G. Garc, Eur. Phys. J. D 44, 125 (2007)

70. H. Zhang, D. Tian, J. Zhao, J. Chem. Phys. 129, 114302 (2008)
71. F. Chen, R.L. Johnston, Acta Mater. 56, 2374 (2008)

72. B. Zhu, G. Thrimurthulu, L. Delannoy, C. Louis, C. Mottet, J. Creuze, B. Legrand, H. Guesmi, J. Catal. 308, $272(2013)$

73. A. Dhouib, H. Guesmi, Chem. Phys. Lett. 521, 98 (2012)

74. K.J. Taylor, C.L. Pettiette-Hall, O. Cheshnovsky, R.E. Smalley, J. Chem. Phys. 96, 3319 (1992)

75. T.H. Lee, K.M. Enin, J. Phys. Chem. 98, 10023 (1994)

76. D. Stolcic, M. Fischer, G. Gantefo, Y.D. Kim, Q. Sun, P. Jena, J. Am. Chem. Soc. 125, 2848 (2003)

77. D. Chan, R. Dietsche, G. Ganteför, Y. Dok, Chem. Phys. Lett. 457, 391 (2008)

78. H. Kahnouji, H. Najafvandzadeh, S.J. Hashemifar, M. Alaei, Chem. Phys. Lett. 630, 101 (2015) 